\title{
Entwicklung der Baumarten im Schweizer Wald - methodische Überlegungen
}

Philippe Duc Urs-Beat Brändli Fabrizio Cioldi Adrian Lanz Ulrich Ulmer
Eidgenössische Forschungsanstalt für Wald, Schnee und Landschaft $(\mathrm{CH}) \dagger$ Eidgenössische Forschungsanstalt für Wald, Schnee und Landschaft $(\mathrm{CH})^{*}$ Eidgenössische Forschungsanstalt für Wald, Schnee und Landschaft $(\mathrm{CH})$ Eidgenössische Forschungsanstalt für Wald, Schnee und Landschaft $(\mathrm{CH})$ Eidgenössische Forschungsanstalt für Wald, Schnee und Landschaft (CH)

\section{Development of tree species in Swiss forests - some methodological considera- tions}

\begin{abstract}
Swiss forests have been subject to more stress in recent decades due to increased climatic and biogenic disturbances. Some tree species, such as Norway spruce, have been more severely affected than others. How the tree species composition of the Swiss forest has changed during this time has been assessed with data from the Swiss National Forest Inventory (NFI). The four indicators, presence, dominance, number of stems and basal area, were examined to see: whether the changes in the most important tree species are significant; whether the indicators have developed in the same way in the two diameter classes, D1 (12-36 cm DBH) and D2 (> $36 \mathrm{~cm}$ $\mathrm{DBH})$; and how different diameter thresholds (12 or $36 \mathrm{~cm} \mathrm{DBH)} \mathrm{and} \mathrm{different} \mathrm{circular} \mathrm{sample} \mathrm{plot} \mathrm{sizes}$ $\left(200 \mathrm{~m}^{2}\right.$ or $\left.500 \mathrm{~m}^{2}\right)$ affect the development and significance of the indicators. All the values were estimated for the $5370 \mathrm{NFI}$ forest plots that were accessible in all three inventories, NFI1 (1983-85), NFI2 (1993-95) and NFI3 (2004-06). Only in a minority of tree species did changes in the presence, dominance, number of stems and basal area develop in the same way. Most indicators for the conifer species spruce, fir and pine decreased significantly, whereas for the broadleaf species, maple and ash, as well as larch and the other conifers, they increased significantly. The basal area increased during the period investigated for all tree species except pine and spruce. The different development of the indicators number of stems and basal area can be attributed to a different development within the DBH classes D1 and D2. The inventory diameter threshold strongly affects the development of the indicator number of stems, but not that of the indicator basal area.
\end{abstract}

Keywords: tree species, significance of changes, presence, dominance, number of stems, basal area, inventory diameter threshold, circular sample plot size, Swiss National Forest Inventory

doi: $10.3188 /$ szf. 2011.0326

*Zürcherstrasse 111, CH-8903 Birmensdorf, E-Mail urs-beat.braendli@wsl.ch

S eit einigen Jahrzehnten ist der Schweizer Wald grösseren Belastungen ausgesetzt, insbesondere wegen der Zunahme von klimatischen und biogenen Störungen (Usbeck et al 2010, Engesser et al 2008). Entsprechend war der Anteil der Zwangsnutzungen an der gesamten Nutzungsmenge in den vergangenen Jahren sehr gross: Seit dem ersten Landesforstinventar (LFI1, 1983-1985) war rund die Hälfte aller waldbaulichen Eingriffe zumindest teilweise auf Zwangsnutzungen zurückzuführen. Die mittlere Zwangsnutzungsmenge stieg von jährlich 2.2 Mio. $\mathrm{m}^{3}$ zwischen LFI1 und LFI2 (Herold \& Stierlin 1999) auf 2.9 Mio. $\mathrm{m}^{3}$ zwischen LFI2 und LFI3 (Schwyzer et al 2010). Infolge vorübergehend höherer Holzpreise war auch die reguläre Holznutzung zwischen dem LFI2 (1993-95) und dem LFI3 (2004-06) relativ gross. Darum war die Menge des genutzten und abgestorbenen Holzes in diesem Zeitraum fast gleich gross wie der Holzzuwachs (Duc et al 2010), im Mittelland gar um 23\% grösser. Ob sich der Wald in der Schweiz infolge der häufiger aufgetretenen Störungen und der intensiveren Holznutzung in seiner Baumartenzusammensetzung verändert hat, kann mit Daten des LFI analysiert werden.

Die Entwicklung der Baumartenzusammensetzung wurde im LFI3-Ergebnisbericht anhand der Veränderung von Stammzahl und Vorrat quantifiziert (Cioldi et al 2010). Für die Beschreibung von Pflanzenpopulationen gibt es weitere Merkmale, die sich als Indikatoren für Veränderungen eignen: etwa Präsenz, Dominanz und Deckungsgrad der Arten, sowie Total und Anteil der Variablen Basalfläche oder Biomasse. Je nach Fragestellung kommen unterschied- 


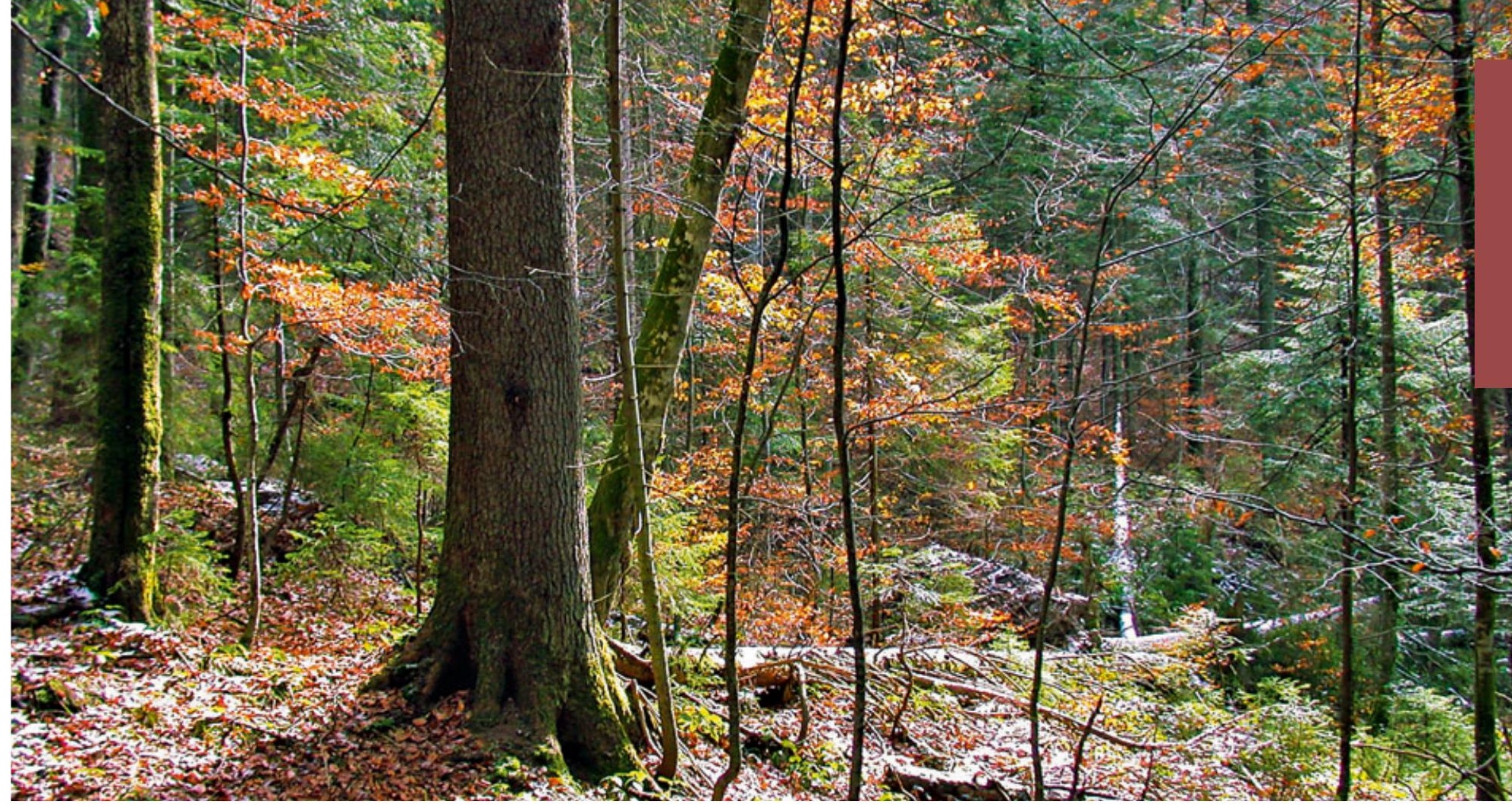

Abb 1 Die Feststellung einer Art auf einem Plot ist auch vom gewählten Indikator, von der Plotgrösse und der Kluppschwelle abhängig. liche Indikatoren zum Einsatz: Präsenz, Dominanz und Biomasse werden primär als ökologische Indikatoren, der Holzvorrat wird als ökonomischer Indikator verwendet. Die Stammzahlverteilung auf Durchmesserklassen ist ein guter Indikator für die Nachhaltigkeit von Baumarten. Die mit dem HolzvorratengkorrelierteBasalfläche(Stammquerschnittsfläche) ist ein Mass für die Bestandesdichte. Da die Basalfläche mit der Kronenfläche eines Baumes korreliert, dient sie auch zur groben Abschätzung des Flächenanteils einer Baumart.

Für die Analyse von Veränderungen eignen sich Merkmale, welche für die Fragestellung relevant sind und reproduzierbar erfasst werden können. Im LFI sind dies insbesondere der Brusthöhendurchmesser (BHD) der Probebäume und alle davon ableitbaren Merkmale. Für unsere Untersuchung haben wir die Indikatoren Präsenz, Stammzahl, Basalfläche und Dominanz ausgewählt. Anhand dieser Indikatoren wird untersucht, ob sich die wichtigsten Baumarten im Schweizer Wald zwischen LFI1 und LFI3 signifikant verändert haben und ob die Veränderungen gleichgerichtet verlaufen sind. Um den zeitlichen Verlauf der Entwicklung aufzuzeigen, werden die Ergebnisse der ersten Periode (LFI1 zu LFI2) mit jenen der zweiten Periode (LFI2 zu LFI3) verglichen.

Das LFI erfasst Probebäume von 12 bis $36 \mathrm{~cm}$ BHD (als Klasse D1 bezeichnet) auf einem Probekreis von $200 \mathrm{~m}^{2}$ und Probebäume ab $36 \mathrm{~cm}$ BHD (Klasse D2) auf einem Kreis von $500 \mathrm{~m}^{2}$. Die Stammzahlentwicklung in den Klassen D1 und D2 könnte unterschiedlich sein und wird im Folgenden für einige Indikatoren getrennt dargestellt.

Das LFI weist mit $12 \mathrm{~cm}$ BHD die höchste Kluppschwelle aller europäischen Länder auf (Tomp- po et al 2009). Ob und wie sich unterschiedliche Kluppschwellen auf Stammzahl- und Basalflächenveränderungen auswirken, wird durch den Vergleich der Entwicklung aller Probebäume (ab $12 \mathrm{~cm}$ BHD) mit jener der Probebäume in der Klasse D2 (ab 36 cm BHD) untersucht.

Für die Berechnung der Hektarwerte pro Probefläche, die sogenannte lokale Dichte (Mandallaz 2008), und letztlich für die Schätzung von Mittelwerten, Totalen und Anteilen der Variablen Stammzahl und Basalfläche in den Auswertungseinheiten der Inventur müssen die Probebäume der Klasse D1, welche auf einer Probefläche von $200 \mathrm{~m}^{2}$ erfasst werden, mit dem Faktor 50 gewichtet werden, jene der Klasse D2, welche auf einer Probefläche von $500 \mathrm{~m}^{2}$ erfasst werden, mit dem Faktor 20 (Köhl 2001). Die Kennzahlen der Grundgesamtheit (Populationsparameter) für Stammzahl und Basalfläche der Klassen D1 und D2 sowie für alle Bäume ab einem BHD von $12 \mathrm{~cm}$ können mithilfe dieser Hochrechnungsfaktoren aus der LFI-Stichprobe unverzerrt geschätzt werden. Nebenbei sei erwähnt, dass alle Kenngrössen auch nur mit den Probebäumen auf dem kleinen Kreis $\left(200 \mathrm{~m}^{2}\right)$ geschätzt werden können; in diesem Fall müssen auch die Bäume mit einem BHD von mehr als $36 \mathrm{~cm}$ mit dem Faktor 50 gewichtet werden.

Während die Indikatoren Stammzahl und Basalfläche, bei richtiger Anwendung der Hochrechnungsfaktoren, nicht von Form und Grösse der verwendeten Probeflächen respektive der Anzahl Probeflächen abhängen, gilt dies nicht für die Indikatoren Präsenz und Dominanz (Abbildung 1). Sie sind als Anteil derjenigen Probeflächen an der Gesamtstichprobe definiert, auf denen eine bestimmte Baumart vorkommt, respektive die sie dominiert, d.h. 
den grössten (lokalen) Basalflächenanteil aufweist. Es ist intuitiv leicht verständlich, dass diese zwei Indikatoren im Allgemeinen von der Grösse (oder Art) der verwendeten Probeflächen als auch vom Stichprobenumfang abhängen. Die so definierten Indikatoren Präsenz und Dominanz lassen sich also nicht (nur sehr bedingt) mit den Ergebnissen anderer National-, Regional- oder Betriebsinventuren vergleichen. Die beobachteten Veränderungen von Präsenz und Dominanz der Baumarten auf dem Netz der permanent angelegten und wiederholt gemessenen LFIProbeflächen lassen sich aber durchaus interpretieren und unterstützen so die vertiefte Analyse der Entwicklung der Baumarten im Schweizer Wald.

\section{Methode}

\section{Berechnung von Zustand und Veränderung} bei permanenten Probeflächen

Das LFI ist eine Kontrollstichprobe, d.h. die Probeflächen im Basisnetz von $1.414 \mathrm{~km} \times 1.414 \mathrm{~km}$ werden wiederholt aufgenommen (Brassel et al 2010). Bisher erfolgte die Erhebung periodisch rund alle zehn Jahre, nämlich erstmals in den Jahren 1983 bis 1985 (LFI1; Mahrer et al 1989), dann 1993 bis 1995 (LFI2; Brassel \& Brändli 1999) und 2004 bis 2006 (LFI3; Brändli 2010). Die terrestrische Inventur setzt sich zusammen aus der Erhebung von Probebäumen ab einer Kluppschwelle von 12 cm BHD auf zwei konzentrischen Kreisflächen und aus der Erfassung von Bestandesdaten auf einer Fläche von $50 \mathrm{~m} \times 50 \mathrm{~m}$. Aus der wiederholten Aufnahme der Probebäume im systematischen Stichprobennetz können unverzerrte Schätzungen für Zustände und Veränderungen aller Variablen (z.B. Stammzahl, Vorrat) für den Schweizer Wald und seine Hauptregionen berechnet werden.

Der Mittelwert $\bar{X}$ einer Plotvariablen $X$ im Untersuchungsgebiet und die Varianz dieses Mittelwerts unter einer einfachen Zufallsstichprobe im Untersuchungsgebiet werden wie folgt geschätzt:

$$
\begin{aligned}
& \hat{\bar{X}}=\frac{1}{n} \sum_{i=1}^{n} X_{i} \\
& \hat{V}(\hat{\bar{X}})=\frac{1}{n} \hat{\sigma}^{2}=\frac{1}{n} \frac{\sum_{i=1}^{n}\left(X_{i}-\hat{\bar{X}}\right)^{2}}{n-1}
\end{aligned}
$$

wobei $\hat{\sigma}^{2}$ die Varianz (Streuung) der einzelnen Plotwerte $X$ im Untersuchungsgebiet und $X_{i}$ den Wert der Variablen $X$ auf dem $i$-ten (von $n$ ) Probeflächen bezeichnet.

Das 95\%-Vertrauensintervall für die Schätzung $\hat{\bar{X}}$ hat bei genügend grossem Stichprobenumfang $n$ etwa die Grenzen:

$$
[\hat{\bar{X}}-1.96 \sqrt{\hat{V}(\hat{\bar{X}})} ; \hat{\bar{X}}+1.96 \sqrt{\hat{V}(\hat{\bar{X}})}]
$$

Im System der verbundenen Stichproben des LFI mit periodischen Wiederholungsmessungen auf den fest eingerichteten Probeflächen (permanente Stichprobe) lassen sich Veränderungen zwischen zwei Zeitpunkten leicht berechnen und mit grosser Genauigkeit schätzen. Es sei $X_{1 . i}$ der Zustandswert der Variablen $X$ auf der Probefläche $i$ zum Zeitpunkt der Vorinventur (oder Erstinventur) und $X_{2 . i}$ der Zustandswert der Variablen $X$ auf der Probefläche $i$ zum Zeitpunkt der Nachinventur (oder Zweitinventur).

Die auf den einzelnen Probeflächen beobachteten Differenzen der Zustandswerte, die Variabe $\delta$ mit Wert

$$
\delta_{i}=X_{2 . i}-X_{1 . i}
$$

für die $i$-te Probefläche, wird als neue Plotvariable für die Schätzung der mittleren Veränderung im Untersuchungsgebiet eingesetzt. Die obigen Formeln 1 bis 3 können analog mit $\delta_{i}$ für $X_{i}$ verwendet werden.

\section{Berechnung der ausgewählten Indikatoren}

Stammzahl und Basalfläche sind metrische Daten, welche aus den Probebäumen des kleinen $\left(200 \mathrm{~m}^{2}\right)$ respektive des kombinierten Probekreises $\left(200 / 500 \mathrm{~m}^{2}\right)$ mithilfe der Hochrechnungsfaktoren für jede Probefläche gerechnet werden. Die mittleren Hektarwerte und ihre Standardfehler werden gemäss Formel 1 und 3 geschätzt; die Veränderungen gemäss Formel 4. Bei Veränderungen kann der Schätzfehler einfach interpretiert werden: Ist die halbe Breite des 95\%-Vertrauensintervalls kleiner als der Absolutwert der geschätzten Veränderung, dann ist diese statistisch signifikant auf dem 95\%-Niveau, d.h., es kann mit grosser Wahrscheinlichkeit davon ausgegangen werden, dass sich die Population zwischen den beiden Inventuren bezüglich der untersuchten Kenngrösse tatsächlich in die geschätzte Richtung verändert hat. Veränderungen werden auch häufig in Prozent des Ausgangswertes angegeben (prozentuale Veränderung), ein Versuch, das Ausmass der Veränderung auf unterschiedlichen Skalen respektive für verschiedene Variablen vergleichbar zu machen.

Die Präsenz von Pflanzenarten ist allgemein abhängig davon, auf welcher Fläche sie erfasst wird (Magurran 2004). Aussagen zur Präsenz der Baumarten müssen sich darum auf einen einheitlichen Probekreis beziehen. Vergleichbare Daten zur Artenpräsenz liegen im LFI für Probebäume ab $12 \mathrm{~cm}$ BHD im kleinen Probekreis vor. Alle Werte zur Präsenz wurden deshalb für den kleinen Kreis $\left(200 \mathrm{~m}^{2}\right)$ berechnet. Artenpräsenz ist ein binäres Probeflächenmerkmal: Die Art kommt vor (1) oder nicht vor (0). Berechnet wird der Anteil (Prozent) der Probeflächen mit Präsenz einer bestimmten Baumart an der Gesamtheit der Probeflächen in der Auswertungseinheit. Ob sich die Präsenz signifikant verändert hat, wird - wie für die metrischen Merkmale Stammzahl 
und Basalfläche - mit der Differenz der binären Werte zwischen Zweit- und Erstaufnahme untersucht.

Als dominante Baumart für eine bestimmte Probefläche wird hier die (Haupt-)Baumart mit der grössten Basalfläche bezeichnet. Sie kann mit allen Probebäumen auf dem kombinierten Probekreis mit entsprechenden Hochrechnungsfaktoren berechnet werden oder - wie in dieser Untersuchung - nur mit den Probebäumen im kleinen Kreis. Wie die Präsenz wird die Dominanz für die zwölf Hauptbaumarten(gruppen) in ein binäres Merkmal überführt, und die statistische Analyse erfolgt mit den weiter vorne angegebenen Formeln.

\section{Festlegung der übrigen Rahmenwerte}

Im LFI werden Veränderungen der Waldfläche auf dem gesamten Stichprobennetz, Veränderungen von Aufbau und Zusammensetzung des Waldes dagegen auf den gemeinsamen Probeflächen der zu vergleichenden Inventuren geschätzt (Brassel et al 2010). Veränderungsauswertungen auf diesen gemeinsamen, zugänglichen Waldprobeflächen weisen gewisse praktische Vorteile auf (Lanz \& Abegg 2010): Die benötigten Informationen liegen vollständig vor, und der Einfluss unterschiedlicher Netze auf die festgestellten Veränderungen kann ausgeschlossen werden. Entsprechend haben wir alle Werte für die gemeinsamen,

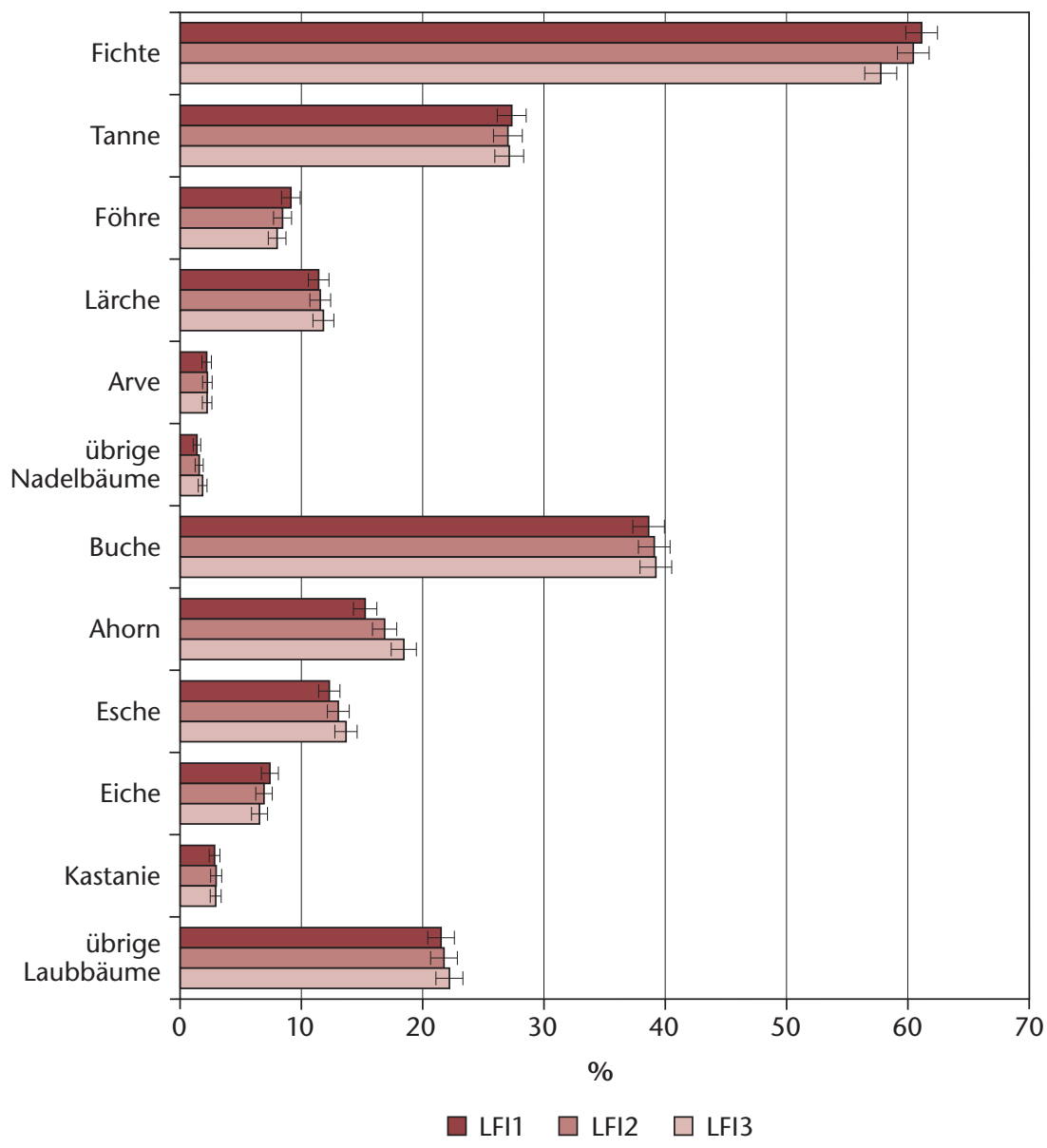

Abb 2 Präsenz nach Baumart und Inventur auf dem 2-Ar-Kreis (Kluppschwelle $12 \mathrm{~cm}$; Signifikanzniveau 95\%). zugänglichen Waldprobeflächen der drei Inventuren LFI1, LFI2 und LFI3 gerechnet. Diese Stichprobe umfasst 5370 Probeflächen im 1.414-km×1.414-km-Netz. Im Unterschied zum LFI-Bericht wurden nicht alle lebenden Bäume, sondern nur die stehend-lebenden Probebäume berücksichtigt. Die stehend-lebenden Probebäume machen im LFI3 91.2\% aller erfassten (lebenden und toten) Probebäume aus.

Im LFI-Bericht werden Ergebnisse zur Artenzusammensetzung überwiegend nach den Baumgattungen Fichte, Tanne, Föhre, Lärche, Arve, Buche, Ahorn, Esche, Eiche, Kastanie ausgewiesen und als Hauptbaumarten bezeichnet. Sie werden ergänzt mit den zwei Sammelklassen «übrige Nadelbäume» und «übrige Laubbäume». Auf die Baumarten Fichte (Picea abies), Tanne (Abies alba), Lärche (Larix decidua), Buche (Fagus sylvatica), Esche (Fraxinus excelsior) und Kastanie (Castanea sativa) hat diese Aggregation kaum Auswirkungen, da die übrigen Arten dieser Gattungen sehr selten sind (Cioldi et al 2010). Die Arve (Pinus cembra), obschon eine Föhrenart, wird seit dem LFI1 als Hauptbaumart geführt. Die Hauptbaumart Föhre vereint neben der Waldföhre (P. sylvestris) auch die baumförmig wachsende Bergföhre (P. mugo) sowie die beiden Exoten Strobe (P. strobus) und Schwarzföhre (P. nigra). Die Hauptbaumart Ahorn vereint Berg- (Acer pseudoplatanus), Spitz(A. platanoides), Feld- (A. campestre) und schneeballblättriger Ahorn (A. opalus), die Hauptbaumart Eiche Stiel- (Quercus robur), Trauben- (Q. petraea), Zerr(Q. cerris) und Flaumeiche (Q. pubescens) sowie die exotische Roteiche (Q. rubra). In unserem Indikatorenvergleich verwenden wir dieselbe Aggregation.

\section{Ergebnisse}

\section{Präsenz}

Bei einer Kluppschwelle von $12 \mathrm{~cm}$ und untersucht auf dem kleinen Kreis $\left(200 \mathrm{~m}^{2}\right)$ kam die Fichte im LFI1 auf 61\%, die Buche auf 39\% aller Probeflächen vor (Abbildung 2). Zwischen LFI1 und LFI3 nahm die Präsenz von Fichte, Föhre und Eiche in der Schweiz markant ab, jene von Ahorn, Esche und den übrigen Nadelbäumen markant zu (Tabelle 1).

Die prozentualen Veränderungen der Präsenz lagen mehrheitlich im Bereich \pm 1 bis $5 \%$. Signifikante Abnahmen der Präsenz gab es bei den Föhren und Eichen in beiden Perioden und bei den Fichten in der zweiten Periode, signifikante Zunahmen bei Ahorn, Esche und den übrigen Nadelbäumen (Tabelle 1).

\section{Dominanz}

Insgesamt dominieren die drei häufigsten Baumarten Fichte, Buche und Tanne auf 70\% des Schweizer Waldes (Abbildung 3). Auf rund 5\% der Probeflächen gibt es keine Probebäume und entspre- 


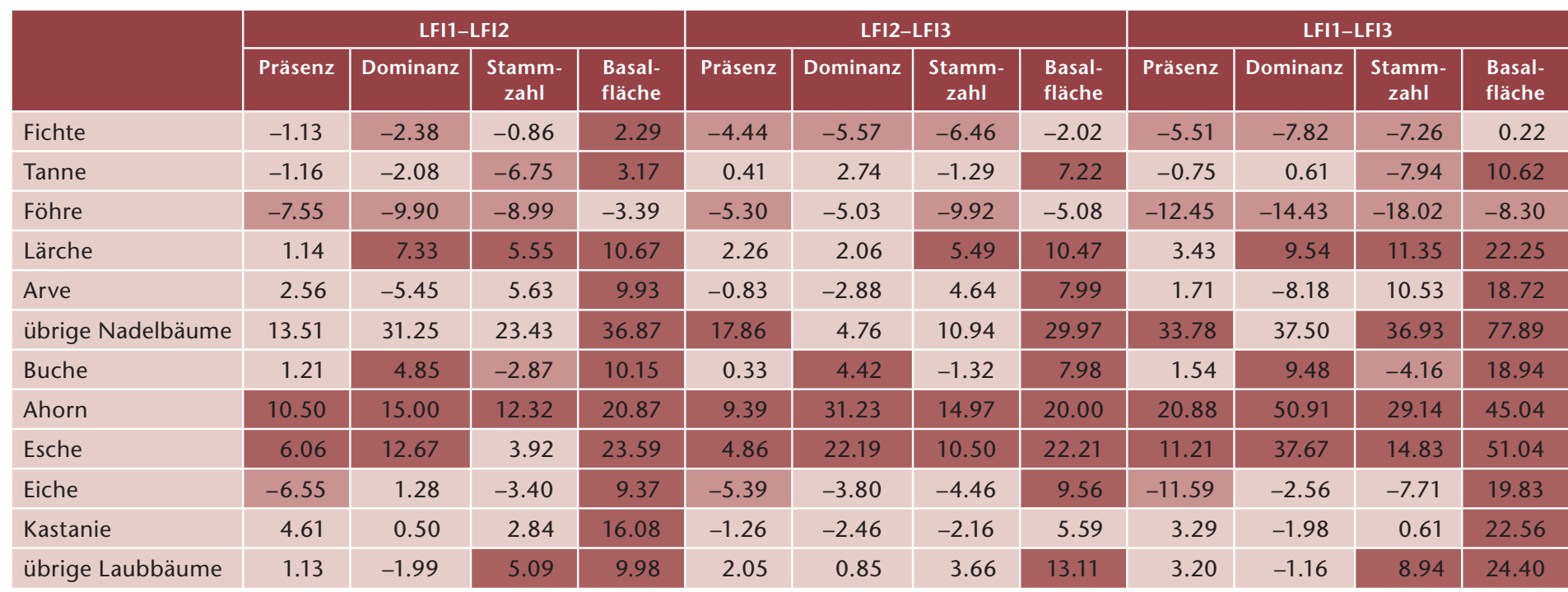

Tab 1 Prozentuale Veränderung der Indikatoren Präsenz, Dominanz, Stammzahl und Basalfläche auf dem 2-Ar-Kreis (Kluppschwelle 12 cm; Signifikanzniveau 95\%). Hervorgehoben sind die Zellen mit einer signifikanten Abnahme (mittlerer Farbton) und jene mit einer signifikanten Zunahme (dunkler Farbton).

chend keine dominierende Baumart. Seit dem LFI1 nahm die Dominanz von Fichte und Föhre deutlich $\mathrm{ab}$, jene von Lärche, Buche, Ahorn und Esche deutlich zu. Im LFI3 dominierten Nadelbäume noch auf rund 60\% der Waldfläche (LFI1: 63\%), Laubbäume auf 35\% (LFI1: 32\%).

Die prozentualen Veränderungen der Dominanz streuten etwas stärker als jene der Präsenz und

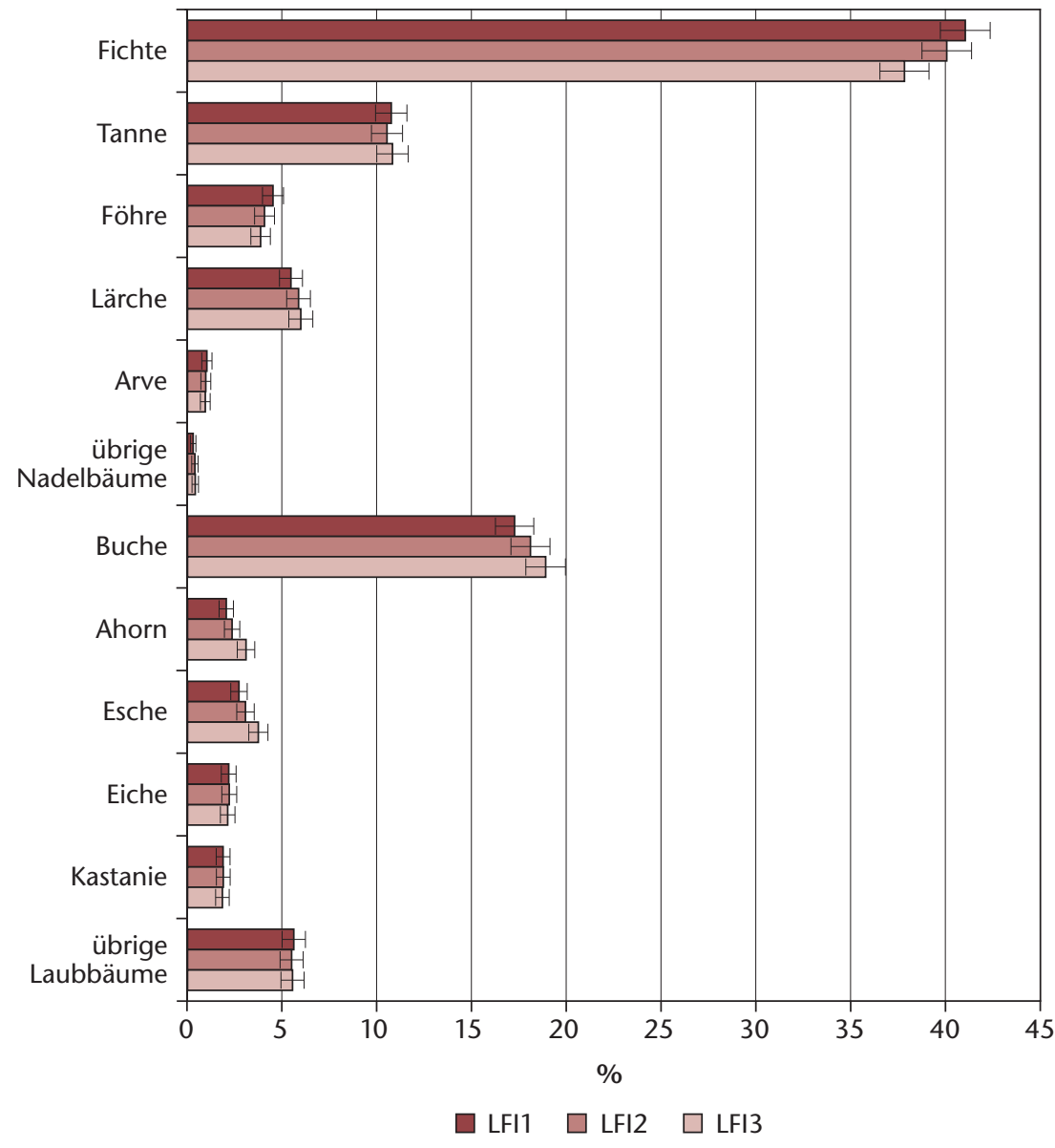

Abb 3 Dominanz nach Baumart und Inventur auf dem 2-Ar-Kreis (Kluppschwelle $12 \mathrm{~cm}$; Signifikanzniveau 95\%). betrugen teilweise über $\pm 10 \%$ (Tabelle 1). In der ersten Periode nahm die Dominanz von Fichte und Föhre signifikant ab, jene von Lärche, Buche, Ahorn und Esche nahm signifikant zu. In der zweiten Periode verlor die Fichte weiter an Dominanz, während Buche und insbesondere Ahorn und Esche kräftig zulegten.

\section{Stammzahl}

Zwischen LFI1 und LFI3 waren die Stammzahlen von Fichte, Tanne, Föhre und Buche signifikant rückläufig, jene von Lärche, Ahorn und Esche sowie der Sammelkategorien übrige Nadelbäume und übrige Laubbäume nahmen deutlich zu (Abbildung 4).

Obwohl die absoluten Stammzahlveränderungen grösstenteils weniger als 2 St./ha ausmachen, sind die Stammzahlabnahmen von Tanne, Föhre und Buche in der ersten Periode, von Fichte und Föhre in der zweiten Periode und von Fichte, Tanne, Föhre und Buche über beide Perioden signifikant. Signifikante Stammzahlzunahmen gab es bei Lärche, Ahorn, Esche (nur in der zweiten Periode und im Vergleich LFI1-LFI3) sowie bei den übrigen Laubbäumen (erste Periode und LFI1-LFI3) und den übrigen Nadelbäumen (nur LFI1-LFI3).

\section{Basalfläche}

Zwischen LFI1 und LFI3 nahm die Basalfläche bei allen Baumarten ausser bei Fichte und Föhre signifikant zu (Abbildung 5), und die Zunahmen waren, mit Ausnahme der Kastanie in der zweiten Periode, auch in den einzelnen Perioden statistisch signifikant (Tabelle 1).

Die Basalflächenabnahme bei der Föhre war in beiden Perioden nicht signifikant, insgesamt zwischen LFI1 und LFI3 dagegen schon. Auffallend ist der Basalflächenrückgang bei der Fichte in der zweiten Periode; dieser Rückgang ist jedoch nur auf dem 68\%-Niveau signifikant, und insgesamt resultiert für 




Abb 4 Stammzahl nach Baumart und Inventur auf dem 2-Ar-Kreis (Kluppschwelle $12 \mathrm{~cm}$; Signifikanzniveau 95\%).

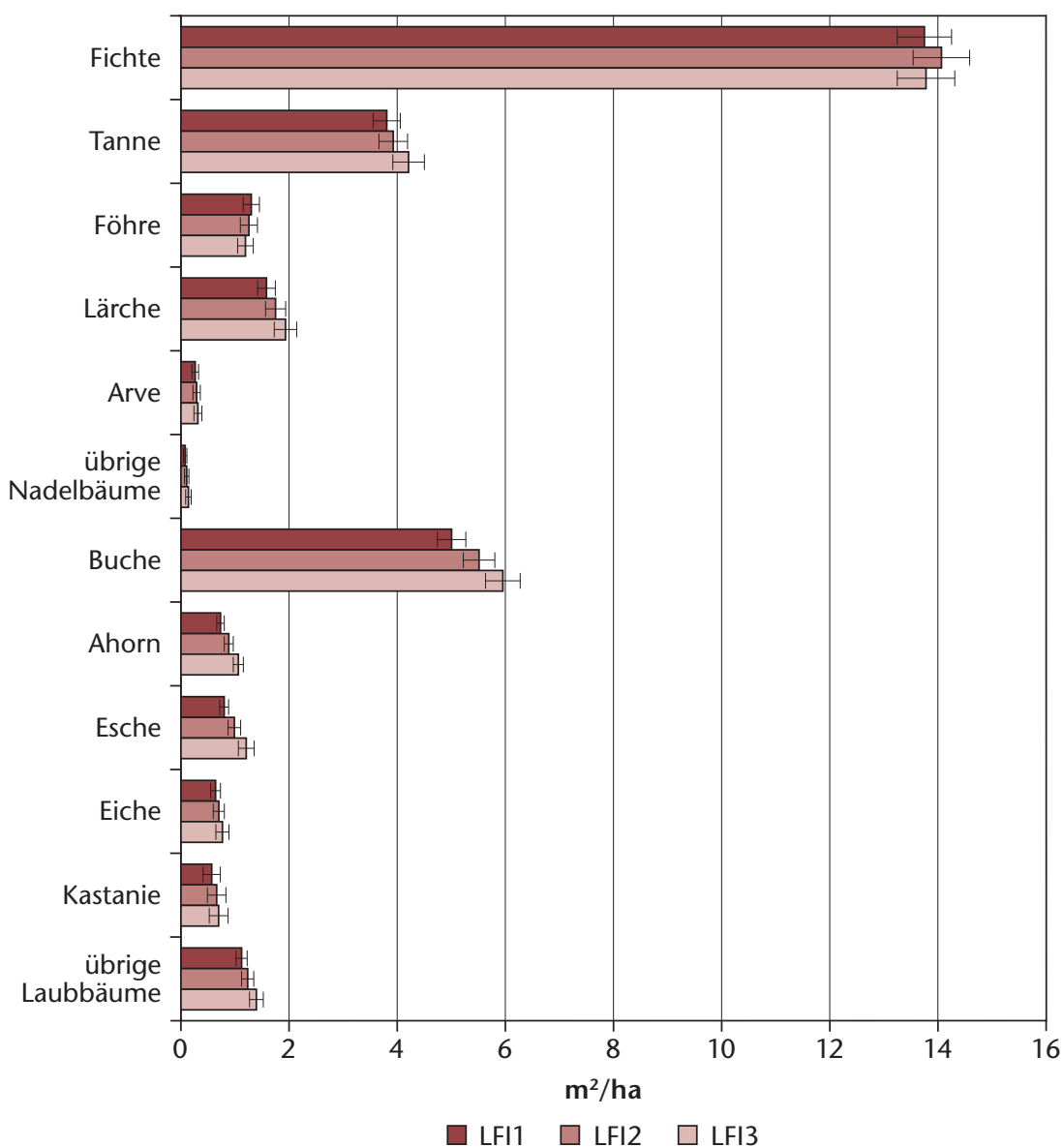

Abb 5 Basalfläche nach Baumart und Inventur auf dem 2-Ar-Kreis (Kluppschwelle $12 \mathrm{~cm}$; Signifikanzniveau 95\%). die Fichte zwischen LFI1 und LFI3 keine signifikante Veränderung.

\section{Vergleich der vier Indikatoren}

Während bei Präsenz, Dominanz und Stammzahl signifikante Zu- und Abnahmen in beiden Perioden in etwa gleich häufig vorkamen, nahm die Basalfläche bei fast allen Baumarten signifikant zu (Tabelle 1). Bei vielen Baumarten verlief die Entwicklung der Indikatoren in den beiden Perioden und insgesamt nicht gleichgerichtet. Zwischen LFI1 und LFI3 beispielsweise gab es bei Tanne, Buche und Eiche je mindestens einen Indikator mit gegenläufig signifikanter Veränderung. Bei Fichte, Arve, Kastanie und den übrigen Laubbäumen gab es gegenläufige, aber nicht signifikante Veränderungen. Bei Lärche und den übrigen Nadelbäumen waren die Veränderungen gleichgerichtet, aber nicht alle auf dem 95\%-Niveau signifikant. Nur bei der Föhre nahmen alle Indikatoren signifikant $a b$, und bei Ahorn und Esche alle signifikant zu.

\section{Vergleich der beiden Durchmesserklassen}

Zwischen LFI1 und LFI3 nahm die Stammzahl der BHD-Klassen D1 (12 bis $36 \mathrm{~cm}$ BHD) bei fünf Baumarten signifikant ab (Fichte, Tanne, Föhre, Buche, Eiche), und bei einer Baumart (Ahorn) nahm sie signifikant zu (Abbildung 6). In der BHD-Klasse D2 (ab $36 \mathrm{~cm}$ BHD) nahm die Stammzahl nur bei einer Baumart (Fichte) signifikant ab, dagegen nahm sie bei neun Baumarten signifikant zu (Tanne, Lärche, Arve, Buche, Ahorn, Esche, Eiche, Kastanie, übrige Laubbäume). Damit resultierten bei Tanne, Buche und Eiche signifikant gegenläufige Stammzahlentwicklungen in den beiden Klassen.

Bei der Basalflächenentwicklung waren die Unterschiede zwischen den Inventuren LFI1 und LFI3 in beiden BHD-Klassen ähnlich wie bei der Stammzahl (Abbildung 7): In der BHD-Klasse D1 nahm die Basalfläche bei vier Baumarten signifikant ab (Fichte, Tanne, Föhre, Buche), bei fünf Baumarten nahm sie signifikant zu (übrige Nadelbäume, Ahorn, Esche, Kastanie, übrige Laubbäume). In der BHD-Klasse D2 gab es zwischen LFI1 und LFI3 nur eine Basalflächenabnahme bei der Föhre, welche aber nicht signifikant ist; die Basalflächenzunahmen hingegen sind für alle elf Baumarten signifikant. Gegenläufige signifikante Basalflächenveränderungen in den BHD-Klassen D1 und D2 treten also nur bei Fichte, Tanne, Föhre und Buche auf.

Bei einer hypothetischen Kluppschwelle von $36 \mathrm{~cm}$ BHD fällt die Bewertung der Stammzahlveränderung zwischen LFI1 und LFI3 bei Tanne, Buche, Eiche und Kastanie anders aus als bei der aktuellen Kluppschwelle von $12 \mathrm{~cm}$ BHD, nämlich kommt es zu einer signifikanten Zunahme statt einer signifikanten Abnahme respektive keiner Veränderung bei der Kastanie (Abbildung 6). Die Beurteilung der Ba- 


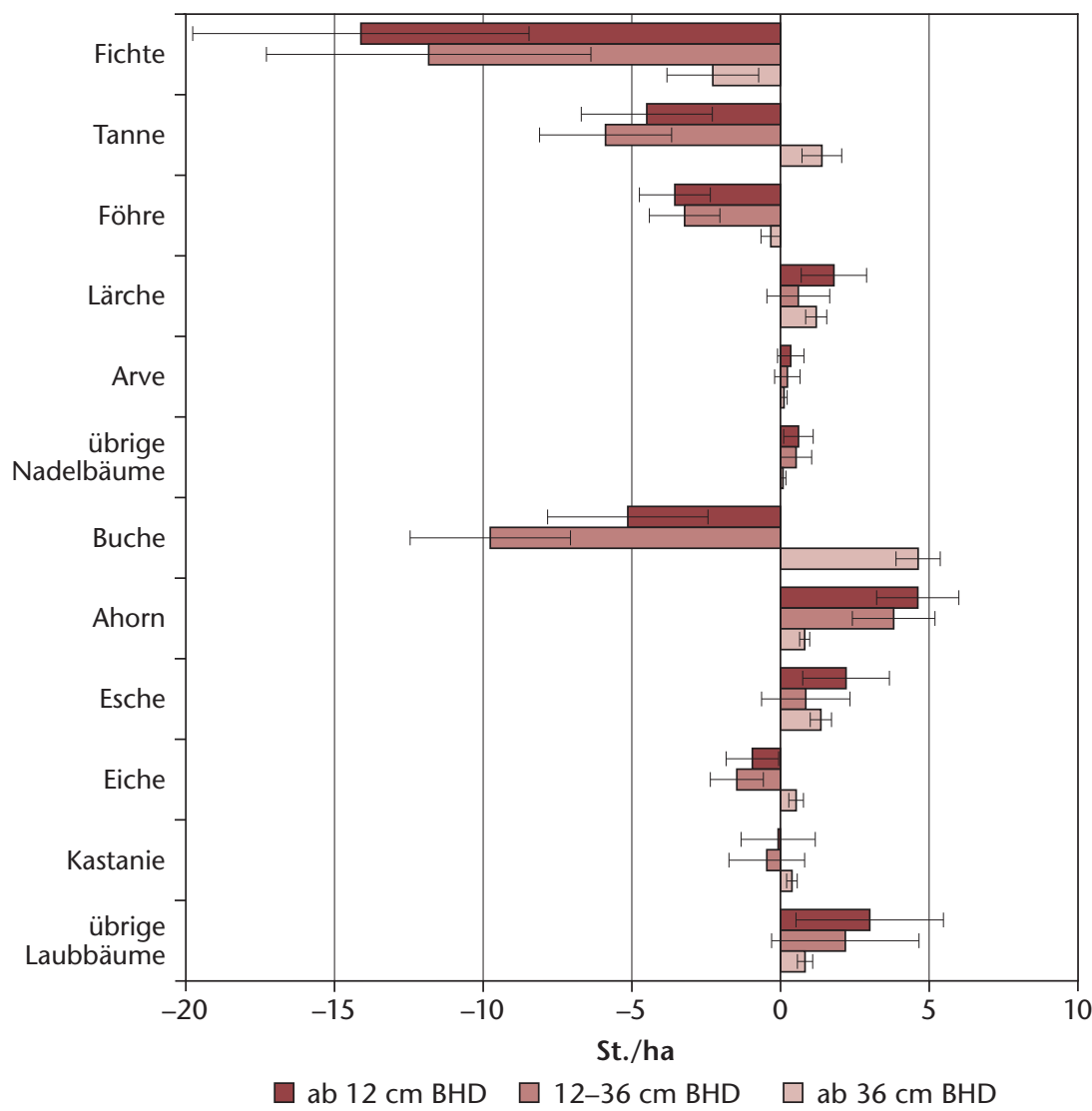

Abb 6 Stammzahlveränderung nach Baumart zwischen LFI1 und LFI3 (kombinierte Kreisflächen). Dargestellt ist die Veränderung aller Bäume (Kluppschwelle $12 \mathrm{~cm} \mathrm{BHD),}$ der Durchmesserklasse D1 (12-36 cm BHD) und der Durchmesserklasse D2 (ab $36 \mathrm{~cm} \mathrm{BHD).}$

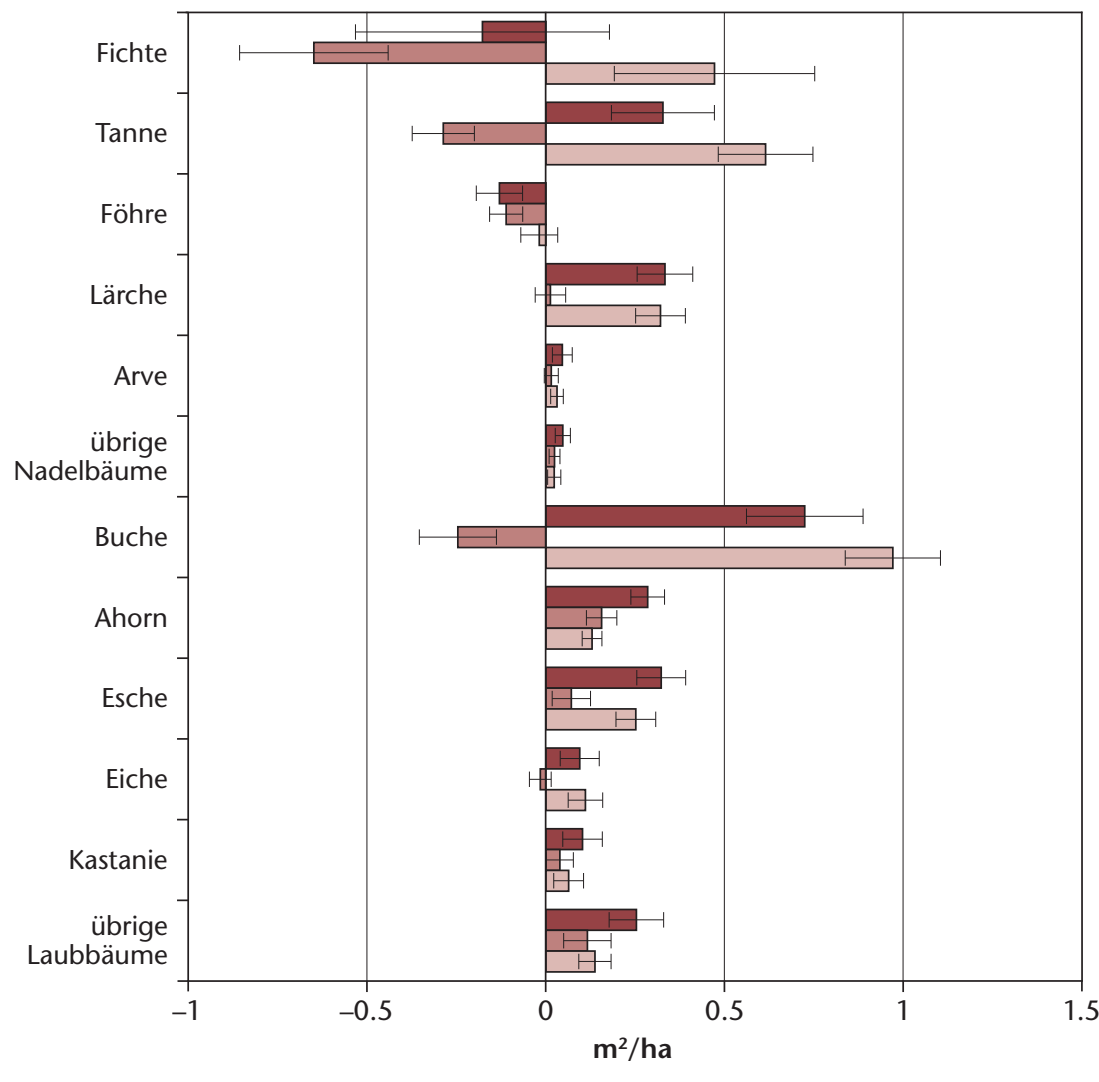

$\square$ ab $12 \mathrm{~cm} \mathrm{BHD} \square$ 12-36 cm BHD $\square$ ab $36 \mathrm{~cm} \mathrm{BHD}$

Abb 7 Basalflächenveränderung nach Baumart zwischen LFI1 und LFI3 (kombinierte Kreisflächen, Signifikanzniveau 95\%). Dargestellt ist die Veränderung aller Bäume (Klupp-

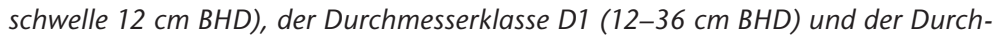
messerklasse D2 (ab $36 \mathrm{~cm} \mathrm{BHD).}$ salflächenveränderung dagegen ist in Klasse D2 praktisch identisch mit derjenigen in der Gesamtpopulation der Bäume ab $12 \mathrm{~cm}$ BHD (Abbildung 7). Einzig bei der Fichte entwickelt sich die Basalfläche in der Klasse D2 (signifikante Zunahme) gegenläufig zur beobachteten Basalflächenabnahme bei einer Kluppschwelle von $12 \mathrm{~cm}$ BHD; allerdings ist die Basalflächenabnahme für die Fichte zwischen LFI1 und LFI3 nicht signifikant. Bei der Föhre ist die Basalflächenabnahme signifikant mit einer Kluppschwelle von $12 \mathrm{~cm}$ und nicht signifikant bei einer Kluppschwelle von $36 \mathrm{~cm}$. Insgesamt wirkt sich eine Verschiebung der Kluppschwelle von $12 \mathrm{~cm}$ auf $36 \mathrm{~cm}$ also primär auf die Stammzahl- und weniger auf die Basalflächenveränderung aus.

\section{Einfluss der Probekreisfläche}

Die Werte für die Präsenz und die Dominanz einer Baumart hängen im Allgemeinen neben der Struktur des Waldes auch von der Grösse der Probeflächen und unter Umständen auch vom Stichprobenumfang ab. In Abbildung 8 ist die Veränderung der Präsenz zwischen LFI1 und LFI3 für die Bäume ab $36 \mathrm{~cm}$ BHD für eine Probeflächengrösse von $200 \mathrm{~m}^{2}$ (kleiner Kreis) und eine Probeflächengrösse von $500 \mathrm{~m}^{2}$ (grosser Kreis) dargestellt. Es ist intuitiv klar, dass eine Baumart in umso mehr Probeflächen vorkommt, je grösser diese sind. Bei der Veränderung der Präsenz stellt man fest, dass mit Ausnahme von Lärche und Arve die beobachteten Veränderungen auf dem grossen Kreis grösser sind als auf dem kleinen Kreis, bei allen Baumarten zeigt die Veränderung (Zunahme oder Abnahme der Präsenz) aber in die gleiche Richtung. Die Veränderung der Präsenz für Bäume in der BHD-Klasse D2 konnte für alle Baumarten sowohl auf dem kleinen wie auf dem grossen Kreis statistisch signifikant nachgewiesen werden respektive für die Kategorie der übrigen Nadelbäume in beiden Fällen nicht nachgewiesen werden.

Ein ähnliches Bild ergibt sich bei der Analyse der Veränderung der Dominanz auf dem kleinen und dem grossen Kreis (Abbildung 9). Die beobachteten Veränderungen der Dominanz zwischen LFI1 und LFI3 zeigen ausser bei der Arve bei allen Baumarten auf dem kleinen und dem grossen Kreis in die gleiche Richtung und sind signifikant respektive auf beiden Kreisen nicht signifikant (Tanne, Arve und Eiche); bei der Kastanie ist die Zunahme der Dominanz signifikant auf dem kleinen Kreis, aber nicht signifikant auf dem grossen Kreis.

\section{Stammzahl und Basalflächenanteil}

Präsenz und Dominanz sind Indikatoren, welche sich auf (unterschiedlich definierte) Baumartenanteile in der Stichprobe abstützen. Zwei weitere Indikatoren, welche in der Praxis häufig verwendet werden, sind die Baumartenanteile an der Gesamtstammzahl und der Gesamtbasalfläche. In Abbil- 




Abb 8 Veränderung der Präsenz auf dem 2-Ar- und dem 5-Ar-Kreis nach Baumart zwischen LFI1 und LFI3 (Kluppschwelle $36 \mathrm{~cm}$, Signifikanzniveau 95\%).



Abb 9 Veränderung der Dominanz auf dem 2-Ar- und dem 5-Ar-Kreis nach Baumart zwischen LFI1 und LFI3 (Kluppschwelle $36 \mathrm{~cm}$, Signifikanzniveau 95\%). dung 10 sind diese vier Indikatoren für die Inventuren LFI1 und LFI3 dargestellt. Beim Vergleich ist zu beachten, dass die Summen der Anteile (Prozente) aller Baumarten bei Stammzahl und Basalfläche bei 100\% liegen, bei der Dominanz wegen der leeren Probeflächen bei etwa 95\% und bei der Präsenz bei über $100 \%$.

Einige modellhafte Überlegungen zu diesen vier Indikatoren helfen, die Verteilung der Baumarten im Schweizer Wald und deren Veränderung besser zu verstehen. Wenn etwa der Schweizer Wald aus lauter Reinbeständen bestünde und alle Baumarten, wenn auch unterschiedlich häufig, mit der identischen Durchmesserverteilung vorkämen, wären die vier Indikatoren für die einzelnen Baumarten jeweils gleich gross. Aber auch sonst haben Baumarten, welche nur in Reinbeständen vorkommen, in Präsenz und Dominanz identische Werte (Kastanie und Arve tendieren dazu).

Baumarten mit verhältnismässig wenig dicken Bäumen haben einen höheren Stammzahl- als Basalflächenanteil, was zurzeit im Schweizer Wald vor allem auf die Laubbäume zutrifft (mit Ausnahme von Kastanie und Eiche). Baumarten mit - im Vergleich zu den anderen drei Indikatoren - hoher Präsenz kommen weniger in Reinbeständen und häufiger gut durchmischt vor; beispielsweise Ahorn, Esche und die übrigen Laubbäume, weniger stark ausgeprägt auch Buche, Eiche, Tanne und die übrigen Nadelbäume, und eher schwach ausgeprägt Lärche, Föhre und Fichte; eine deutliche Tendenz zum Reinbestand haben demnach Kastanie und Arve.

Baumarten mit verhältnismässig vielen dicken Bäumen haben einen höheren Wert beim Basalflächenanteil als bei der Dominanz, unabhängig von ihrer Durchmischung, was etwa für Fichte und Tanne zutrifft. Schliesslich: Je stärker die Baumarten durchmischt sind, desto mehr liegt die Summe der Präsenzen bei über 100\%.

Die Abnahme von Stammzahl-, Basalflächenanteil und Dominanz bei gewissen Baumarten über die Zeit muss bei anderen Baumarten kompensiert werden. Zwischen LFI1 und LFI3 lässt sich dieser Effekt für den Basalflächenanteil und die Dominanz der Nadelbäume beobachten, welche zugunsten der jeweiligen Anteile der Laubbäume abgenommen haben (weniger ausgeprägt bei den Stammzahlanteilen).

\section{Diskussion}

Für alle vier ausgewählten Indikatoren der Baumartenveränderung gibt es geeignete statistische Verfahren zur Berechnung von Veränderungen und zur Prüfung der Signifikanz dieser Veränderungen. Bei den Indikatoren Präsenz, Dominanz und Stammzahl kamen Zu- und Abnahmen in beiden Perioden ungefähr gleich häufig vor. Die Basalfläche dagegen nahm unabhängig von den übrigen Indikatoren bei 

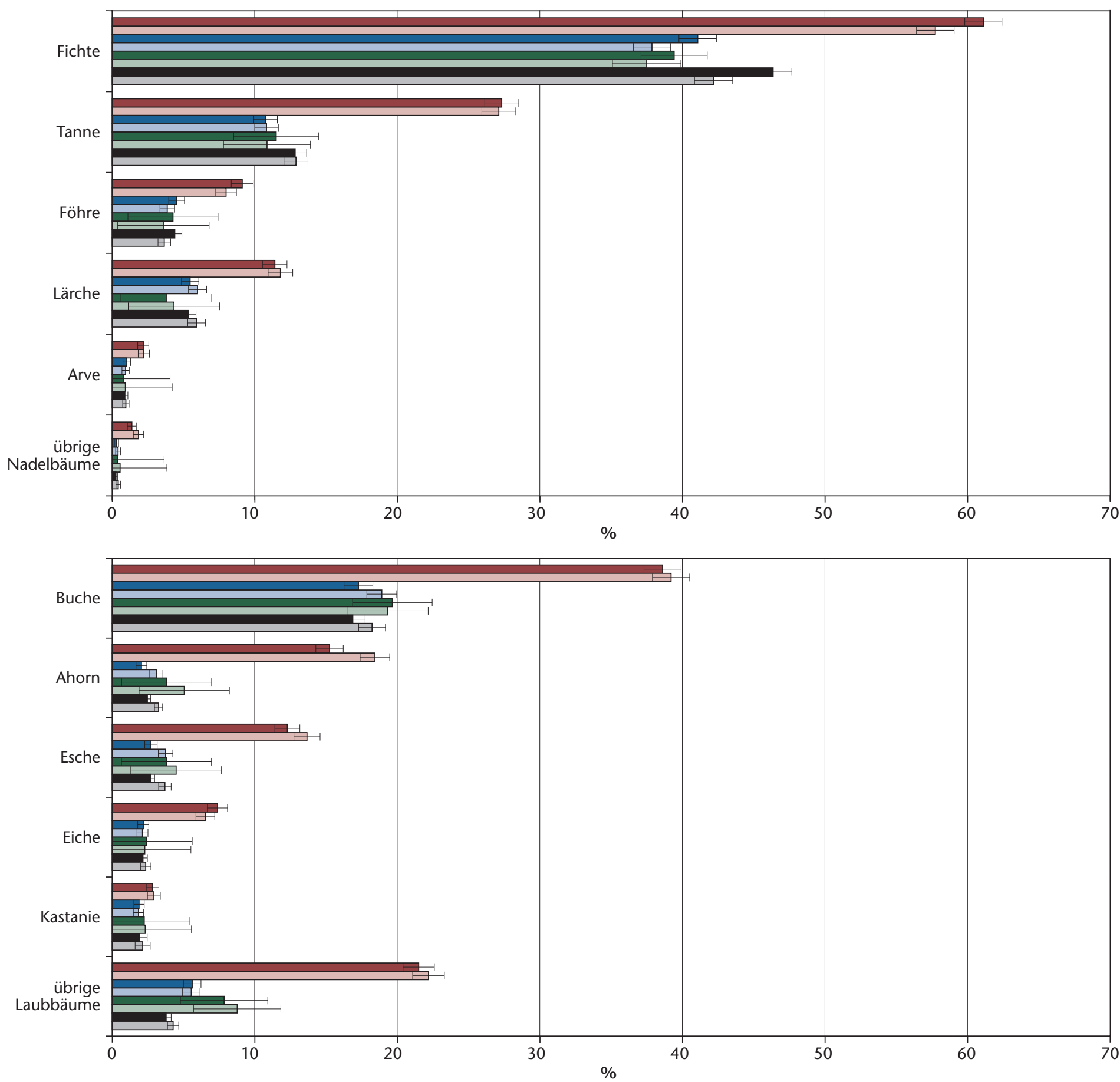

Präsenz LFI1 $\square$ Präsenz LFI3 $\square$ Dominanz LFI1 $\square$ Dominanz LFI3

Stammzahl LFI1 $\square$ Stammzahl LFI3

Basalfläche LFI1 $\square$ Basalfläche LFI3

Abb 10 Präsenz, Dominanz, Stammzahlanteil und Basalflächenanteil auf dem 2-Ar-Kreis nach Baumart im LFI1 und LFI3 (Kluppschwelle 12 cm, Signifikanzniveau 95\%).

fast allen Baumarten zu. Bei neun der zwölf untersuchten Baumarten entwickeln sich die Indikatoren unterschiedlich, wenn neben der Richtung der Veränderung auch die Signifikanz berücksichtigt wird. Nur bei Föhre (Abnahme), Ahorn und Esche ( $\mathrm{Zu}$ nahme) war die Entwicklung aller Indikatoren in der Periode LFI1 bis LFI3 gleichgerichtet und signifikant. Allgemeingültige Aussagen anhand eines einzigen Indikators sind folglich nicht möglich.

Die stark unterschiedliche Entwicklung der Stammzahlen in den Durchmesserklassen D1 und D2 wirkt sich offensichtlich auf die Indikatoren Stammzahl und Basalfläche aus. Mit der Entwick- lung des Schweizer Waldes hin zu vorratsreicheren, durchschnittlich älteren Beständen (Stierlin \& Ulmer 1999, Cioldi et al 2010) verschob sich die Durchmesserverteilung der meisten Baumarten in den letzten zwei Jahrzehnten hin zu dickeren Bäumen. Insgesamt führt dies zu einer Reduktion der mittleren Stammzahlen bei weiter zunehmenden Basalflächen und Vorräten. Bei der Verwendung der Indikatoren für Nachhaltigkeitsbetrachtungen ist diesem Phänomen Rechnung zu tragen.

Bei der Analyse von Veränderungen spielt der Zeitbezug eine Rolle, auch wenn die festgestellten Veränderungen der ersten und der zweiten Periode 
nicht wesentlich verschieden waren. Insbesondere bei der Fichte gibt es zwischen der ersten und zweiten Betrachtungsperiode recht grosse Unterschiede. Die Fichte wurde in der zweiten Periode durch den Sturm Lothar im Winter 1999, den nachfolgenden Borkenkäferbefall und die Trockenheit des Sommers 2003 stark geschädigt (Forster et al 2008) und auch aus wirtschaftlichen Gründen stark genutzt. Entsprechend hat sie bei allen Indikatoren ausser der Basalfläche teilweise markant abgenommen, insbesondere im Mittelland. Natürlicherweise langsam ablaufende Waldentwicklungen wie die Baumartenveränderungen können also durch extreme Naturereignisse und starke Holznutzungen erheblich beschleunigt werden.

Der Einfluss der Kluppschwelle auf Zustand und Entwicklung von Stammzahl und Präsenz ist evident. Deutlich robuster reagieren die Basalfläche und die daraus auf Probeflächen abgeleitete Dominanz der Baumarten auf eine Änderung der Kluppschwelle. Die Schweiz weist mit $12 \mathrm{~cm}$ BHD die höchste Kluppschwelle aller europäischen Länder auf. Um ein vollständiges Bild über die Waldentwicklung zu erhalten, müssten Präsenz und Stammzahl im gesamten Durchmesserbereich bis hinunter auf $0 \mathrm{~cm}$ ausgewiesen werden. Entsprechende Daten werden im LFI seit der Ersterhebung für Bäumchen zwischen 0 und $12 \mathrm{~cm}$ BHD auf sogenannten JungwaldProbekreisen erhoben. Zudem wird die Präsenz aller Gehölzarten, also inklusive der Sträucher, seit dem LFI3 auf dem kleinen Probekreis von $200 \mathrm{~m}^{2}$ erhoben. Im Rahmen des LFI4 sind entsprechende Veränderungsauswertungen möglich.

Während Stammzahl und Basalfläche sowie Stammzahlanteil und Basalflächenanteil auf den kombinierten Probeflächen ausgewertet und leicht mit den Ergebnissen in anderen, regionalen oder internationalen Inventuren verglichen werden können, sind Präsenz und Dominanz der Baumarten auf den Probeflächen abhängig von der Grösse der Probefläche. Zu Vergleichszwecken müssen diese Indikatoren deshalb auf einem Probekreis von einheitlicher Grösse abgeleitet werden. Weil die Angaben zu den Gehölzen bis $36 \mathrm{~cm}$ BHD nur für den kleinen Kreis vorliegen, kommt dafür im LFI nur dieser infrage.

Im LFI wurden Veränderungen überwiegend auf dem 1.414-km×1.414-km-Netz im gemeinsamen, zugänglichen Wald zweier aufeinanderfolgender Inventuren gerechnet (Brassel et al 2010). Dieses Vorgehen ist angezeigt, wenn qualitative Veränderungendes Waldaufbausund der Artenzusammensetzung betrachtet werden. Die ausgewiesenen Veränderungen sind aber dadurch nicht kompatibel mit der Differenz der jeweiligen Inventurzustände (Lanz \& Abegg 2010). Zudem werden die Veränderungen auf den neu eingewachsenen Probeflächen ausser Acht gelassen. Beim Vergleich von quantitativen Merkmalen wie der Artenverbreitung, der Stammzahl oder dem Holzvorrat werden darum im LFI oftmals die Zustände für das Waldareal in den jeweiligen Inventuren dargestellt.

Die bestehende Aggregation der Baumarten zu Hauptbaumarten ist optimiert für Aussagen zur Holzproduktion. Für ökologische Fragestellungen müssten die Auswertungen möglichst nach Baumarten getrennt erfolgen. Die Hauptbaumart Föhre müsste beispielsweise in Waldföhre und Bergföhre unterteilt werden. Statistisch signifikante Veränderungen können selbst bei sehr kleinen Ausgangsstammzahlen nachgewiesen werden. Relevant und interpretierbar sind diese Veränderungen aber erst ab einer Stichprobe von rund 50 Probebäumen, denn darunter können zufällige Effekte eine grosse Rolle spielen. Für 33 Baumarten wird dieser minimale Stichprobenumfang insgesamt erreicht, nicht aber in den einzelnen Regionen. Regionale Aussagen zu den Baumartenveränderungen kann das LFI für die zehn häufigsten Baumarten Fichte, Tanne, Waldföhre, Lärche, Buche, Traubeneiche, Bergahorn, Esche und in eingeschränktem Masse für Mehlbeere und Ulme machen. Arten wie die Arve, die Bergföhre, die Vogelbeere (alle in den Alpen) die Hängebirke und die Kastanie (auf der Alpensüdseite) sind nur in einer einzigen Produktionsregion stark verbreitet und könnten dort ebenfalls differenzierter ausgewertet werden.

Eingereicht: 29. März 2011, akzeptiert (mit Review): 30. Juni 2011

\section{Literatur}

BRÄNDLI UB, EDITOR (2010) Schweizerisches Landesforstinventar. Ergebnisse der dritten Erhebung 2004-2006. Birmensdorf: Eidgenöss Forsch.anstalt Wald Schnee Landschaft. $312 \mathrm{p}$.

BRASSEL P, BRÄNDLI UB, EDITORS (1999) Schweizerisches Landesforstinventar. Ergebnisse der Zweitaufnahme 19931995. Bern: Haupt. 442 p.

BRASSEL P ET AL (2010) Das Landesforstinventar. In: Brändli UB, editor. Schweizerisches Landesforstinventar. Ergebnisse der dritten Erhebung 2004-2006. Birmensdorf: Eidgenöss Forsch.anstalt Wald Schnee Landschaft. pp. 11-29.

CIOLDI F ET AL (2010) Waldressourcen. In: Brändli UB, editor. Schweizerisches Landesforstinventar. Ergebnisse der dritten Erhebung 2004-2006. Birmensdorf: Eidgenöss Forsch. anstalt Wald Schnee Landschaft. pp. 31-113.

DUC P ET AL (2010) Holzproduktion. In: Brändli UB, editor. Schweizerisches Landesforstinventar. Ergebnisse der dritten Erhebung 2004-2006. Birmensdorf: Eidgenöss Forsch. anstalt Wald Schnee Landschaft. pp. 265-288.

ENGESSER R, FORSTER B, MEIER F, WERMELINGER B (2008) Forstliche Schadenorganismen im Zeichen des Klimawandels. Schweiz Z Forstwes 159: 344-351. doi: 10.3188.szf/ 2008.344

FORSTER B, MEIER F, BRÄNDLI UB (2008) Deutlicher Rückgang der Fichte im Mittelland - Vorratsabbau auch durch Sturm und Käfer. Wald Holz 89 (3): 52-54.

HEROLD A, STIERLIN HR (1999) Waldzustand. In: Brassel P, Brändli UB, editors. Schweizerisches Landesforstinventar. Ergebnisse der Zweitaufnahme 1993-1995. Bern: Haupt. pp. 185-231. 
KÖHL M (2001) Inventory concept NFI2. In: Brassel P, Lischke H, editors. Swiss National Forest Inventory: Methods and models of the second assessment. Birmensdorf: Swiss Federal Research Institute. pp. 19-44.

LANZ A, ABEGG M (2010) Erfassung, Schätzung und Analyse von Veränderungen der Waldressourcen im Schweizerischen Landesforstinventar (LFI). In: Römisch K, Wunn U, editors. 21. Tagung der Sektion Forstliche Biometrie und Informatik (Tharandt, 21-22 Sep 2009). Trippstadt: Forschungsanstalt Waldökologie Forstwirtschaft RheinlandPfalz. pp 160-172.

MAGURRAN A (2004) Measuring biological diversity. Malden: Blackwell. $256 \mathrm{p}$.

MAHRER F ET AL (1988) Schweizerisches Landesforstinventar. Ergebnisse der Erstaufnahme 1982-1986. Birmensdorf: Eidgenöss Forsch.anstalt Wald Schnee Landsch, Ber 305. $375 \mathrm{p}$.
MANDALLAZ D (2008) Sampling techniques for forest inventories. Boca Raton: Chapman Hall. 256 p.

SCHWYZER A, ABEGG M, KELLER M, BRANG P (2010) Gesundheit und Vitalität. In: Brändli UB, editor. Schweizerisches Landesforstinventar. Ergebnisse der dritten Erhebung 2004-2006. Birmensdorf: Eidgenöss Forsch.anstalt Wald Schnee Landschaft. pp. 31-113.

STIERLIN HR, ULMER U (1999) Waldaufbau. In: Brassel P, Brändli UB, editors. Schweizerisches Landesforstinventar. Ergebnisse der Zweitaufnahme 1993-1995. Bern: Haupt. pp. 103-150.

TOMPPO E, GSCHWANDTNER T, LAWRENCE M, MCROBERTS R, EDITORS (2010) National forest inventories. Heidelberg: Springer. $607 \mathrm{p}$.

USBECK T ET AL (2010) Increasing storm damage to forests in Switzerland from 1858 to 2007. Agric For Meteorol 150: 47-55.

\section{Entwicklung der Baumarten im Schweizer Wald - methodische Überlegungen}

Seit einigen Jahrzehnten ist der Schweizer Wald wegen der Zunahme von klimatischen und biogenen Störungen grösseren Belastungen ausgesetzt. Gewisse Baumarten wie die Fichte sind davon stärker betroffen als andere. Ob sich die Baumartenzusammensetzung des Schweizer Waldes in diesem Zeitraum verändert hat, wird mit den Daten des Schweizerischen Landesforstinventars (LFI) überprüft. Dazu wird für die vier Indikatoren Präsenz, Dominanz, Stammzahl und Basalfläche untersucht, ob die Veränderungen der wichtigsten Baumarten signifikant sind, ob sich die Indikatoren in zwei Durchmesserklassen D1 (12 bis $36 \mathrm{~cm}$ Brusthöhendurchmesser [BHD]) und D2 (ab $36 \mathrm{~cm}$ BHD) gleichgerichtet entwickelt haben und wie sich unterschiedliche Kluppschwellen (12 vs. $36 \mathrm{~cm}$ BHD) sowie verschieden grosse Probekreisflächen $\left(200 \mathrm{~m}^{2}\right.$ vs. $\left.500 \mathrm{~m}^{2}\right)$ auf die Entwicklung und Signifikanz der Indikatoren auswirken. Alle Werte wurden für die im LFI1 (1983-85), LFI2 (1993-95) und LFI3 (2004-06) gemeinsam zugänglichen Waldprobeflächen $(N=5370)$ berechnet. Veränderungen der Präsenz, Dominanz, Stammzahl und Basalfläche verlaufen nur bei einer Minderheit der Baumarten gleichgerichtet. Bei den Nadelbaumarten Fichte, Tanne und Föhre nahmen die meisten Indikatoren signifikant ab, bei den Laubbaumarten Ahorn und Esche sowie bei der Lärche und den übrigen Nadelbäumen signifikant zu. Die Basalfläche nahm im Untersuchungszeitraum bei allen Baumarten ausser Föhre und Fichte zu. Die unterschiedliche Entwicklung von Stammzahl und Basalfläche ist auf die unterschiedliche Entwicklung in den BHD-Klassen D1 und D2 zurückzuführen. Die Kluppschwelle wirkt sich recht stark auf die Entwicklung der Stammzahl aus, nicht aber auf jene der Basalfläche.

\section{Evolution des essences dans la forêt suisse - considérations méthodologiques}

Depuis quelques décennies, la forêt suisse est exposée à d'importants facteurs stressants dus à l'augmentation des perturbations d'ordre climatique et biotique. Certaines essences, comme l'épicéa, sont plus gravement touchées que d'autres. Afin de savoir si la composition des essences de la forêt suisse s'est modifiée durant cette période, nous avons examiné les données de l'Inventaire forestier national suisse (IFN). La présence des arbres, leur dominance, le nombre de tiges et la surface terrière sont les quatre indicateurs retenus. Ils ont permis d'étudier si les modifications des essences principales sont significatives, si les indicateurs ont évolué dans le même sens à l'intérieur des classes de diamètre D1 (12 à $36 \mathrm{~cm}$ de DHP) et D2 (plus de $36 \mathrm{~cm}$ de DHP), et comment les différents seuils d'inventaire (12 vs. $36 \mathrm{~cm}$ DHP) et surfaces des placettes $\left(200 \mathrm{~m}^{2}\right.$ vs. $\left.500 \mathrm{~m}^{2}\right)$ influencent l'évolution et la signifiance des indicateurs. Toutes les valeurs ont été calculées pour les placettes accessibles communes $(\mathrm{N}=5370)$ lors des IFN1 (1983-85), IFN2 (1993-95) et IFN3 (2004-06). La présence des essences, la dominance, le nombre de tiges et la surface terrière n'évoluent que pour une minorité d'essences dans le même sens. Parmi les résineux, la plupart des indicateurs révèlent une régression significative pour l'épicéa, le sapin et le pin, alors que cette tendance est généralement inversée pour les autres résineux ainsi que pour les feuillus, tels l'érable, le frêne et le mélèze. La surface terrière a augmenté durant la période d'observation pour toutes les essences, excepté le pin et l'épicéa. Les variations du nombre de tiges et de la surface terrière sont dues à l'évolution différente dans les classes de DHP D1 et D2. Le seuil d'inventaire influence assez fortement l'évolution du nombre de tiges, mais pas celle de la surface terrière. 